\title{
Commentary: alpha-synuclein interacts with SOD1 and promotes its oligomerization
}

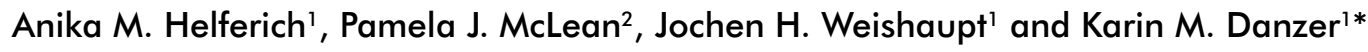 \\ 'Department of Neurology, Ulm University, Albert-Einstein-Allee 11, 89081 Ulm, Germany \\ ${ }^{2}$ Mayo Clinic, Jacksonville, Florida, USA
}

Article Info

\section{Article Notes}

Received: September 22, 2016

Accepted: October 17, 2016

\section{*Correspondence:}

Jun.-Prof. Dr. Karin M Danzer

Ulm University, Department of Neurology, Albert-Einstein-Allee

11, 89081 Ulm, Germany

Telephone: +4973150063049

Fax: +4973150063050

Email: karin.danzer@uni-ulm.de

(c) 2016 Danzer KM. This article is distributed under the terms of the Creative Commons Attribution 4.0 International License

\section{ABSTRACT}

Alpha-synuclein and $\mathrm{Cu}, \mathrm{Zn}$ superoxide dismutase (SOD1) are both aggregation-prone proteins that are associated with Parkinson's disease (PD) and amyotrophic lateral sclerosis (ALS), respectively. Recently, we showed that alpha-synuclein interacts with SOD1 in various cell types and tissues. Using a cell culture model, we also found that alpha-synuclein nucleates the polymerization of SOD1. Here, we discuss the current literature regarding their interaction and their co-localization in aggregates of human post-mortem tissue. Furthermore we comment on the reported alpha-synuclein-induced SOD1 polymerization in terms of cross-seeding effects in neurodegeneration.

\section{Commentary}

Recently, we presented evidence for a so far unknown interaction of alpha-synuclein and SOD1 with potential pathophysiological relevance $^{1}$. Abnormal protein deposits of alpha-synuclein characterize synucleinopathies, a group of neurodegenerative diseases including PD, dementia with Lewy bodies and multiple system atrophy, whereas SOD1 is associated with the pathogenesis of ALS. Mutations in SOD1 lead to ALS and protein aggregates containing SOD1 in a subset of ALS patients.

Our recent study indicates that alpha-synuclein and SOD1 influence each other's aggregation tendency. Using a protein complementation assay, alpha-synuclein was found to increase SOD1 dimerization. Although homodimers of SOD1 are enzymatically active, alpha-synuclein does not seem to change the enzyme activity of SOD $1^{1}$. Alpha-synuclein might induce the formation of either inactive SOD1 dimers or higher molecular aggregates of SOD1 which are thought to be enzymatically inactive ${ }^{2}$. Additional support for an influencing role of alpha-synuclein on the aggregation property of SOD1 comes from the study by Koch et al. ${ }^{3}$. Here, the authors demonstrated that preformed recombinant alpha-synuclein fibrils added to the cell culture media of SOD1 transfected cells induced the polymerization of SOD1. The same was also observed in vivo when recombinant preformed alpha-synuclein fibrils were injected in the temporal cortex of SOD1 ${ }^{\mathrm{G} 93 \mathrm{~A}}$ transgenic mice. These animals presented a considerably elevated number of SOD1 aggregates compared to $\mathrm{NaCl}$ injected animals using immunohistochemical analysis of brainstem sections ${ }^{3}$.

Together, accumulating evidence from in vitro and in vivo 
studies suggests that alpha-synuclein accelerates SOD1 aggregation. However, it still needs to be clarified which molecular SOD1 species are promoted by alpha-synuclein.

The induction of SOD1 dimerization/oligomerization by alpha-synuclein might be based on a prion-like crossseeding activity of alpha-synuclein. Cross-seeding or heterologous seeding describes a pathological process where a misfolded protein acts as a template for the aggregation of a different protein ${ }^{4}$. There are several examples of a heterologous seeding activity of alphasynuclein on other proteins. For instance, alpha-synuclein promotes the polymerization of tau into fibrils, a hallmark of Alzheimer's disease (AD) ${ }^{5,6}$. Whether alpha-synuclein accelerates amyloid beta (abeta) plaque formation, a further neuropathological characteristic of $\mathrm{AD}$, is controversial. In vitro data show an induction of abeta aggregation by alpha-synuclein ${ }^{7}$. In contrast, a more recent study detected decreased abeta deposits due to injection of alpha-synuclein containing homogenates or preformed alpha-synuclein fibrils in hippocampus of an AD mouse model ${ }^{8}$. However, cross-seeding activity of alpha-synuclein has been hypothesized to be the molecular consequence of alpha-synuclein inclusions in $60 \%$ of $\mathrm{AD}$ patients ${ }^{4,9}$.

If alpha-synuclein nucleates SOD1 polymerization due to cross-seeding activity, one could speculate that alpha-synuclein and SOD1 co-aggregate in the same protein deposits, or at least alpha-synuclein and SOD1 are simultaneously present in inclusions of ALS or PD patients. Beside Helferich et al., there are only a few studies showing co-localization of alpha-synuclein and SOD1 in protein aggregates ${ }^{1}$. For instance, inclusions containing both phosphorylated alpha-synuclein and SOD1 were detected in post-mortem CNS tissue of an ALS patient carrying SOD1 mutation (Cys111Tyr) ${ }^{10}$. Nishiyama et al. reported that alpha-synuclein aggregates in the substantia nigra and locus coeruleus of five PD patients were immuno-positive for SOD $1^{11}$. However, these findings should be interpreted with caution because cells without alpha-synuclein inclusions in the substantia nigra and locus coeruleus did not show SOD1 immunoreactivity in this study although SOD1 is known to be expressed in the substantia nigra which has been confirmed by Steinacker et al. ${ }^{12}$. A systematic study with post-mortem material from a large number of patients is needed to determine the frequency and relevance of alpha-synuclein-SOD1 co-localization in protein deposits. Furthermore, alpha-synuclein inclusions are not a common pathological feature of ALS but have been reported by several independent studies ${ }^{10,13-17}$. Coan and Mitchell show that 5 of $46(11 \%)$ ALS post-mortem tissue samples have alphasynuclein inclusions ${ }^{13}$. Additionally, the most frequently used SOD $1^{\mathrm{G} 93 \mathrm{~A}}$ transgenic ALS mouse model by Gurney et al. displays increased alpha-synuclein immunoreactivity in the $\mathrm{CNS}^{18}$. As alpha-synuclein pathology is found only in a minority of ALS brains, alpha-synuclein might be a cofactor for ALS pathology, especially for SOD1 aggregation, besides many other factors. Furthermore alpha-synuclein could also be important for ALS pathogenesis without forming prominent aggregates itself.

Apart from cross-seeding, alpha-synuclein might induce SOD1 dimerization/oligomerization by alternative mechanism including overstraining the ubiquitinproteasome system, inducing cellular stress, and/or perturbing relevant signal cascades. Furthermore, alphasynuclein could promote polymerization of SOD1 by destabilizing the SOD1 conformation. SOD1 monomer binds one copper ion at its active site, needed for the catalytic activity, and one zinc ion that contributes to the stability of SOD $1^{19}$. As alpha-synuclein is also able to bind metallic cations ${ }^{20}$ but does not change SOD1 enzyme activity ${ }^{1}$, alpha-synuclein may preferentially bind zinc ion over copper ion of SOD1 influencing the stability of SOD1.

In conclusion, emerging evidence points to interplay of alpha-synuclein and SOD1 influencing each other's aggregation properties. Whether alpha-synuclein binds SOD1 monomers, dimers or aggregates and whether SOD1 binds membrane-bound alpha-synuclein or truncated alpha-synuclein is currently not known. Nevertheless, we suggest that the role of alpha-synuclein in ALS (especially mutant SOD1-mediated) might have been underestimated and needs further investigation. In this context, it would also be useful to know if alpha-synuclein induces polymerization of other ALS related aggregation-prone proteins like TAR DNA-binding protein 43 (TDP-43) or fused in sarcoma (FUS). Potential therapeutics affecting aggregation formation of one aggregation-prone protein might also have global benefits for other neurodegenerative diseases.

\section{Acknowledgment}

This work was supported by NINDS NS074730.

\section{References}

1. Helferich AM, Ruf WP, Grozdanov V, Freischmidt A, Feiler MS, Zondler L, et al. $\alpha$-synuclein interacts with SOD1 and promotes its oligomerization. Molecular neurodegeneration. 2015; 10: 66.

2. Saccon RA, Bunton-Stasyshyn RK, Fisher EM, Fratta P. Is SOD1 loss of function involved in amyotrophic lateral sclerosis? Brain. 2013; 136: 2342-2358.

3. Koch Y, Helferich AM, Steinacker P, Oeckl P, Walther P, Weishaupt JH, et al. Aggregated alpha-Synuclein Increases SOD1 Oligomerization in a Mouse Model of Amyotrophic Lateral Sclerosis. Am J Pathol. 2016; 186: $2152-2161$

4. Morales R, Moreno-Gonzalez I, Soto C. Cross-seeding of misfolded proteins: implications for etiology and pathogenesis of protein misfolding diseases. PLoS pathogens. 2013; 9(9):e1003537.

5. Giasson BI, Forman MS, Higuchi M, Golbe LI, Graves CL, Kotzbauer PT, et al. Initiation and synergistic fibrillization of tau and alphasynuclein. Science. 2003; 300(5619): 636-640. 
6. Guo JL, Covell DJ, Daniels JP, Iba M, Stieber A, Zhang B, et al. Distinct $\alpha$-synuclein strains differentially promote tau inclusions in neurons. Cell. 2013; 154: 103-117.

7. Ono K, Takahashi R, Ikeda T, Yamada M. Cross-seeding effects of amyloid $\beta$-protein and $\alpha$-synuclein. Journal of neurochemistry. 2012; 122(5): 883-890.

8. Bachhuber T, Katzmarski N, McCarter JF, Loreth D, Tahirovic S, Kamp F, et al. Inhibition of amyloid- $\beta$ plaque formation by $\alpha$-synuclein. Nat Med. 2015; 21(7): 802-807.

9. Hamilton RL. Lewy bodies in Alzheimer's disease: a neuropathological review of 145 cases using alpha-synuclein immunohistochemistry. Brain pathology (Zurich, Switzerland). 2000; 10(3): 378-384.

10. Takei Y, Oguchi K, Koshihara H, Hineno A, Nakamura A, Ohara S. $\alpha$-Synuclein coaggregation in familial amyotrophic lateral sclerosis with SOD1 gene mutation. Hum Pathol. 2013; 44(6): 1171-1176.

11. Nishiyama K, Murayama S, Shimizu J, Ohya Y, Kwak S, Asayama K. Cu/ Zn superoxide dismutase-like immunoreactivity is present in Lewy bodies from Parkinson disease: a light and electron microscopic immunocytochemical study. Acta neuropathologica. 1995; 89(6): 471-474.

12. Steinacker P, Berner C, Thal DR, Attems J, Ludolph AC, Otto M. Protease-resistant SOD1 aggregates in amyotrophic lateral sclerosis demonstrated by paraffin-embedded tissue (PET) blot. Acta neuropathologica communications. 2014; 2: 130.

13. Coan G, Mitchell CS. An Assessment of Possible Neuropathology and Clinical Relationships in 46 Sporadic Amyotrophic Lateral Sclerosis Patient Autopsies. Neurodegener Dis. 2015; 15(5): 301-312.
14. Cooper-Knock J, Frolov A, Highley JR, Charlesworth G, Kirby J, Milano A, et al. C9ORF72 expansions, parkinsonism, and Parkinson disease: a clinicopathologic study. Neurology. 2013; 81(9): 808-811.

15. Doherty MJ, Bird TD, Leverenz JB. Alpha-synuclein in motor neuron disease: an immunohistologic study. Acta neuropathologica. 2004; 107(2): 169-175.

16. Takeda T, Iijima M, Uchihara T, Ohashi T, Seilhean D, Duyckaerts C. TDP-43 Pathology Progression Along the Olfactory Pathway as a Possible Substrate for Olfactory Impairment in Amyotrophic Lateral Sclerosis. Journal of neuropathology and experimental neurology. 2015; 74(6): 547-556.

17. Yamada T, Itoh K, Matsuo K, Yamamoto Y, Hosokawa Y, Koizumi T, et al. Concomitant alpha-synuclein pathology in an autopsy case of amyotrophic lateral sclerosis presenting with orthostatic hypotension and cardiac arrests. Neuropathology. 2014; 34(2): 164-169.

18. Chung YH, Joo KM, Kim MJ, Cha CI. Immunohistochemical study on the distribution of alpha-synuclein in the central nervous system of transgenic mice expressing a human $\mathrm{Cu} / \mathrm{Zn}$ superoxide dismutase mutation. Neuroscience letters. 2003; 342(3): 151-154.

19. Forman HJ, Fridovich I. On the stability of bovine superoxide dismutase. The effects of metals. J Biol Chem. 1973; 248(8): 26452649.

20. Lashuel HA, Overk CR, Oueslati A, Masliah E. The many faces of $\alpha$-synuclein: from structure and toxicity to therapeutic target. Nature reviews. Neuroscience. 2013; 14(1): 38-48. 